\title{
THE NONEXISTENCE OF A FACTORIZATION FORMULA FOR CAYLEY NUMBERS
}

\author{
by P. J. C. LAMONT
}

(Received 27 October, 1981)

Let $C$ be the Cayley algebra defined over the real field. If, for given elements $\alpha, \beta$, and $\gamma$ of a quaternion subalgebra of $C, \alpha=\beta \gamma$, it follows, by associativity, that for any nonzero element $\delta$ of the same quaternion subalgebra, $\alpha=(\beta \delta)\left(\delta^{-1} \gamma\right)$. For Cayley numbers $\zeta$, $\xi$, and $\eta$ with $\zeta=\xi \eta$, the relation $\zeta=(\xi \delta)\left(\delta^{-1} \eta\right)$ in general only holds when $\delta$ is a nonzero real number. Because of the existence of factorization results $[\mathbf{1}, 2]$ in the orders of $C$, the question naturally arises of whether it is possible to choose one-to-one mappings, $\theta$ and $\phi$, of $C$ onto itself such that $\zeta=\theta \xi$. $\phi \eta$ whenever $\zeta=\xi \eta$. To discuss this question, we make the following definition.

An ordered triple of one-to-one mappings $(\theta, \phi, \psi)$ of $C$ onto itself is defined to be an isotopism of $C$ if

$$
\theta \xi . \phi \eta=\psi \xi \eta \text { for all } \xi, \eta \text { in } C .
$$

An isotopism in which the mappings $\theta, \phi, \psi$ denote multiplication by reals is called trivial. For example, the isotopism $(\iota,-\iota,-\iota)$ where $\iota$ is the identity mapping and $-\iota$ maps every element onto its negative is a trivial isotopism of $C$.

The identity

$$
u[(\alpha \beta) u]=(u \alpha)(\beta u)
$$

in $C$ provides a convenient example of an isotopism of $C$ which is nontrivial.

The triple of mappings $(\theta, \phi, \iota)$ of $C$ upon itself, if an isotopism, is called a principal isotopism of $C$. We prove

THEOREM. There does not exist a nontrivial principal isotopism of $C$.

Proof. Suppose that $(\theta, \phi, \iota)$ is a principal isotopism of $C$. Let $i_{s}(s=0,1, \ldots, 7)$ be as usual the basic units of $C$. Write

$$
\theta i_{s}=u_{s}, \phi i_{s}=w_{s} \text { for } 0 \leq s \leq 7 .
$$

Then the sets $u_{s}, w_{s}$ for $s=0,1, \ldots, 7$ are not necessarily units of $C$. Let $N w_{s}=c_{s}$. Then $u_{s} w_{t}=\theta i_{s}, \phi i_{t}=i_{s} i_{t}$ and $u_{0} w_{0}=i_{0} i_{0}=1$. Hence

$$
c_{0} u_{0}=\bar{w}_{0}
$$

Also $u_{0} w_{t}=i_{0} i_{t}=i_{\mathrm{t}}$ and $u_{t} w_{0}=i_{t} i_{0}=i_{t}$. Therefore

$$
c_{0} u_{t}=i_{t} \bar{w}_{0}
$$

Glasgow Math. J. 24 (1983) 131-132. 
For $1 \leq t \leq 7$, we have $u_{t} w_{t}=i_{t} i_{t}=-1$. Thus

$$
c_{t} u_{t}=-\bar{w}_{t} \text {. }
$$

For $1 \leq s \leq 7, u_{t} w_{s}=i_{t} i_{s}$ and therefore by (4) we have

$$
c_{s} u_{t}=\left(i_{t} i_{s}\right) \bar{w}_{s}=-\left(i_{t} i_{s}\right) c_{s} u_{s}
$$

Hence

$$
u_{\mathrm{t}}=-\left(i_{\mathrm{t}} i_{\mathrm{s}}\right) u_{\mathrm{s}}
$$

It follows by (2) and (3) that for $1 \leq t \leq 7$

$$
c_{0} u_{t}=i_{\imath} \bar{w}_{0}=c_{0} i_{\imath} u_{0}
$$

Thus

$$
u_{t}=i_{t} u_{0}
$$

By (5) and (6) for $1 \leq t, s \leq 7$

$$
i_{t} u_{0}=-\left(i_{t} i_{s}\right) u_{s}=-\left(i_{t} i_{s}\right)\left(i_{s} u_{0}\right) .
$$

Now let $u_{0}=\xi_{0}+\xi_{1} v$ where $\xi_{0}, \xi_{1}$ are quaternions in $\left(i_{t}, i_{s}, i_{t} i_{s}\right)$ and $v$ is another unit different from \pm 1 . We assume that $t \neq s$. Then the right hand side of (7) equals

$$
\begin{aligned}
-\left(i_{t} i_{s}\right)\left(i_{s} \xi_{0}+i_{s}\left(\xi_{1} v\right)\right) & =-\left(i_{t} i_{s}\right)\left(i_{s} \xi_{0}+\left(\xi_{1} i_{s}\right) v\right) \\
& =i_{t} \xi_{0}-\left(i_{1} i_{s}\right)\left(\left(\xi_{1} i_{s}\right) v\right) \\
& =i_{t} \xi_{0}-\left(\xi_{1} i_{t}\right) v \\
& =i_{t} \xi_{0}-i_{t}\left(\xi_{1} v\right) \\
& =i_{t}\left(\xi_{0}-\xi_{1} v\right) .
\end{aligned}
$$

But the left hand side of (7) equals $i_{t}\left(\xi_{0}+\xi_{1} v\right)$. Thus $\xi_{0}+\xi_{1} v=\xi_{0}-\xi_{1} v$. Therefore $\xi_{1}=0$. Now $i_{t}, i_{s}$ can be chosen as any pair of units of $C$. Thus $u_{0}$ is real. The result follows by (2), (3) and (4). i.e. all principal isotopisms of Cayley's algebra $C$ are trivial.

\section{REFERENCES}

1. P. J. C. Lamont, Factorization and arithmetic functions for orders in composition algebras, Glasgow Math. J. 14 (1973), 86-95.

2. R. A. Rankin, A certain class of multiplicative functions, Duke Math. J. 13 (1946), 281-306.

Quantitative and Information Science Department

WESTERN ILLINOIS UNIVERSITY

MACOMB, ILLINOIS 61455

U.S.A. 